\title{
Saint-Sébastien, 1876-1931
}

Naissance d'une capitale touristique

\section{Maitane Ostolaza}

\section{(2) OpenEdition}

Journals

Édition électronique

URL : http://journals.openedition.org/agedor/1558

DOI : 10.4000/agedor. 1558

ISSN : 2104-3353

Éditeur

Laboratoire LISAA

Référence électronique

Maitane Ostolaza, «Saint-Sébastien, 1876-1931 », L'Âge d’or [En ligne], 10 | 2017, mis en ligne le 14 janvier 2019, consulté le 02 mai 2019. URL : http://journals.openedition.org/agedor/1558; DOI :

10.4000/agedor.1558

Ce document a été généré automatiquement le 2 mai 2019.

L'Âge d'or. Images dans le monde ibérique et ibéricoaméricain 


\title{
Saint-Sébastien, 1876-1931
}

\author{
Naissance d'une capitale touristique
}

\section{Maitane Ostolaza}

Dans le cadre du « tournant spatial » que l'historiographie a connu ces dernières années ${ }^{1}$, il semble pertinent d'analyser les phénomènes qui ont marqué la contemporanéité des pays ibéro-américains, en prenant l'espace urbain comme objet d'étude. Comme l'ont souligné les organisateurs du colloque au cours duquel la première version de cet article a été présentée ${ }^{2}$, il est nécessaire d'aborder l'analyse des morphologies urbaines dans la sphère hispanique dans une optique comparatiste, ce en tenant compte également de leur évolution historique. Selon l'argument qui sous-tend cette rencontre, la ville est définie comme un espace complexe, un carrefour espace-temps qui reflète la multiplicité des fonctions, des paysages et des cultures qui caractérisent des sociétés en constante mutation. Cependant, la ville n'est pas seulement le décor passif de ces changements, elle a aussi participé activement à la construction et à la redéfinition des identités collectives contemporaines.

2 À partir de ces prémisses, j'aimerais aborder une étude de cas, celle de la ville de SaintSébastien à la fin du $\mathrm{XIX}^{\mathrm{e}}$ siècle et au début du $\mathrm{xx}^{\mathrm{e}}$ siècle. Mon objectif est d'analyser comment un phénomène complexe comme le tourisme a servi à «inventer » une ville moderne dans laquelle convergent de multiples identités sociales et culturelles.

Le développement du tourisme, en tant que phénomène de masse, est lié aux transformations économiques et sociales qui accompagnent l'industrialisation. Bien qu'en principe il s'agisse d'une activité concernant les élites, à partir du début du $\mathrm{xx}^{\mathrm{e}}$ siècle, elle s'est progressivement étendue aux classes moyennes et populaires.

4 Le tourisme a été analysé en tant que ressource économique ou comme une industrie génératrice d'emplois et de richesses ${ }^{3}$. Il a également été abordé en relation avec la société de masse et les nouvelles habitudes de loisirs qui y sont liées ${ }^{4}$. Par ailleurs, les spécialistes de l'histoire du tourisme distinguent trois niveaux d'approche du sujet : celui relevant de la demande sociale ou des types de tourisme; celui qui prend en compte les facteurs qui relèvent de l'offre, à partir de la conception du tourisme comme une industrie (transport, hébergement et promotion); et, enfin, les études qui se focalisent 
sur les professionnels du secteur, y compris l'administration publique et les agents privés ${ }^{5}$

Dans ce travail, les différents niveaux d'analyse sont pris en compte, d'une manière ou d'une autre, bien que l'accent soit mis sur la figure du touriste, principal vecteur des changements produits dans la ville de Saint-Sébastien à la suite du développement du tourisme. Il s'agira de voir dans quelle mesure les pratiques sociales des touristes contribuent à façonner un nouveau paysage urbain tout en renforçant l'« agentivité » ( agentivity) de ce paysage dans les processus de formation des identités collectives contemporaines ${ }^{6}$.

\section{L'« invention » d'une ville touristique}

6 La pertinence de l'exemple choisi tient au fait que Saint-Sébastien est une ville qui inaugure le «tourisme de plage » moderne tant au Pays Basque qu'en Espagne. D'autre part, Saint-Sébastien et, par extension, la côte basque, qui bénéficiait du prestige de la capitale du Guipúzcoa en tant que destination touristique, ont connu une nouvelle dynamique culturelle et identitaire au début $d u x^{e}$ siècle, marquée en grande partie par le tourisme ${ }^{7}$.

7 Il faut rappeler que la province de Guipúzcoa faisait déjà partie des destinations thermales fréquentées par les élites espagnoles depuis le début du XIX ${ }^{e}$ siècle ${ }^{8}$. En outre, les principales stations thermales de la région (Cestona, Alzola) ont hébergé des complexes hôteliers et des installations de loisirs qui ont servi de précédent aux pratiques touristiques associées aux «bains de mer $»^{9}$. Sur cette base, et à partir du milieu du XIX ${ }^{e}$ siècle, une série de facteurs ont convergé pour placer Saint-Sébastien parmi les destinations touristiques espagnoles et européennes les plus en vogue à l'époque. Tout d'abord, ses conditions naturelles et climatiques attirent l'élite espagnole, de plus en plus séduite par les charmes du littoral ${ }^{10}$. Deuxièmement, le discours hygiéniste du XIX ${ }^{e}$ siècle fait l'éloge des bienfaits des bains de mer dans les eaux froides, ce qui met en valeur les "plages du Nord »" Enfin, la convergence des intérêts politiques et économiques locaux jette les bases de la modernisation de la ville, favorisant la création d'infrastructures et d'équipements urbains qui s'avèrent essentiels pour l'essor du tourisme ${ }^{12}$. Parmi les facteurs politiques il convient de souligner la décision des autorités provinciales d'installer définitivement la capitale de Guipúzcoa à Saint Sébastien (1854), la démolition des remparts et la construction de l'Ensanche à partir de 1863, due à l'architecte Cortázar, la libéralisation du voyage sur le territoire national une fois l'exigence du passeport obligatoire éliminée (Décret Royal du 17 décembre 1863) ou l'insertion de la ville dans la ligne ferroviaire Madrid-Irún, au détriment d'autres capitales voisines comme Bilbao ou Pampelune. 


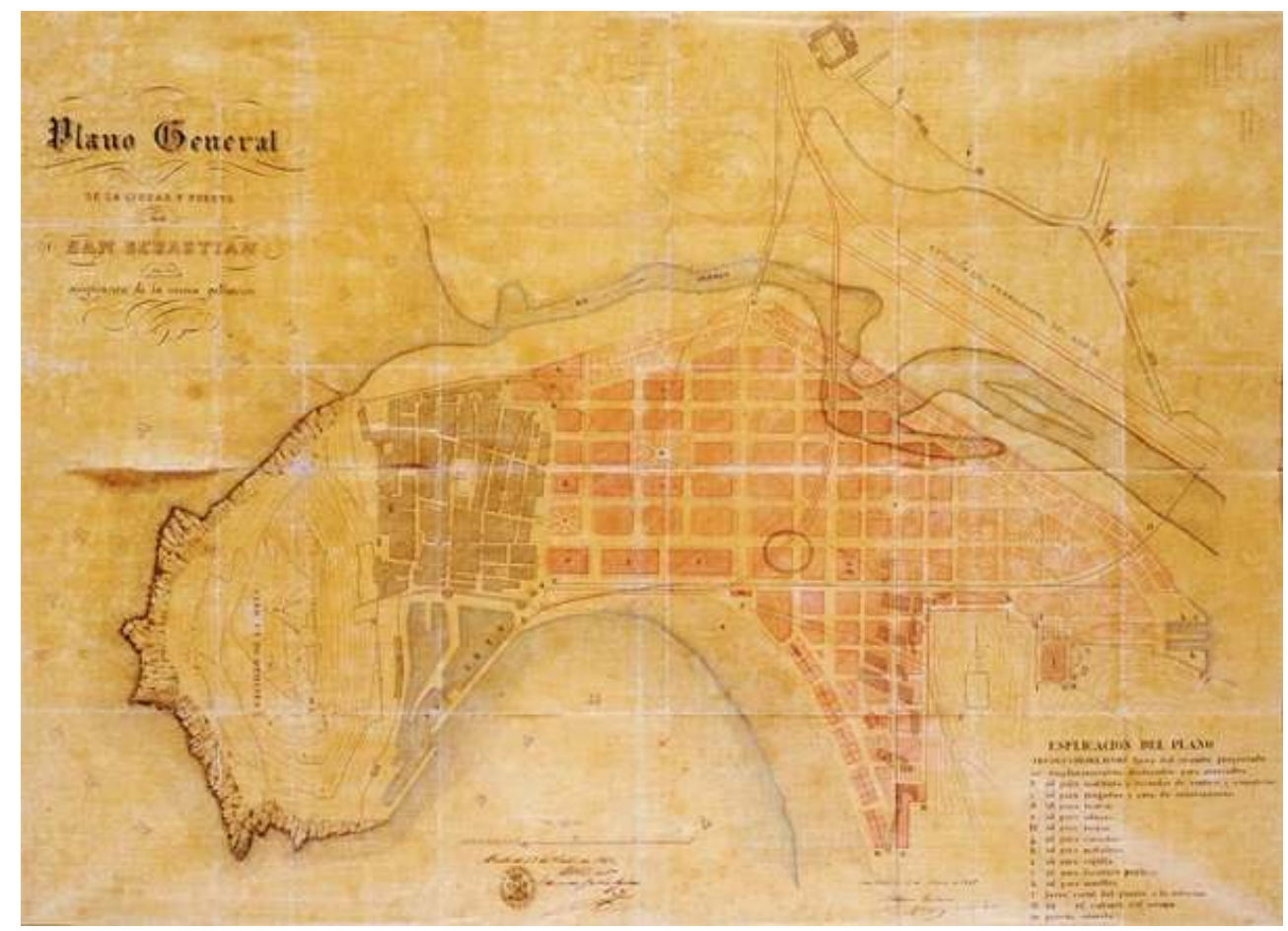

Archives municipales de Saint-Sébastien

Néanmoins, le décollage du tourisme à Saint-Sébastien pourrait difficilement s'expliquer sans tenir compte de la convergence de deux autres éléments : d'une part, le choix de Saint-Sébastien comme résidence d'été par la famille royale espagnole (la reine Isabelle II en 1845 et la reine Régente María Cristina, depuis 1887), ce qui entraîna un effet de mode incontestable tout en attirant le tourisme d'élite vers la capitale basque; et, d'autre part, le pari des autorités municipales en faveur du tourisme. Les bases pour la création d'un véritable «système touristique » dans la ville ont ainsi été jetées ${ }^{13}$.

De plus, l'activité touristique, outre le fait de provoquer un effet d'entraînement sur l'économie locale, aura un impact culturel significatif en contribuant à la formation d'un imaginaire collectif dans lequel les ingrédients de la culture locale, nationale et européenne s'entremêlent dans des proportions variables, en fonction du contexte et des circonstances politiques. 


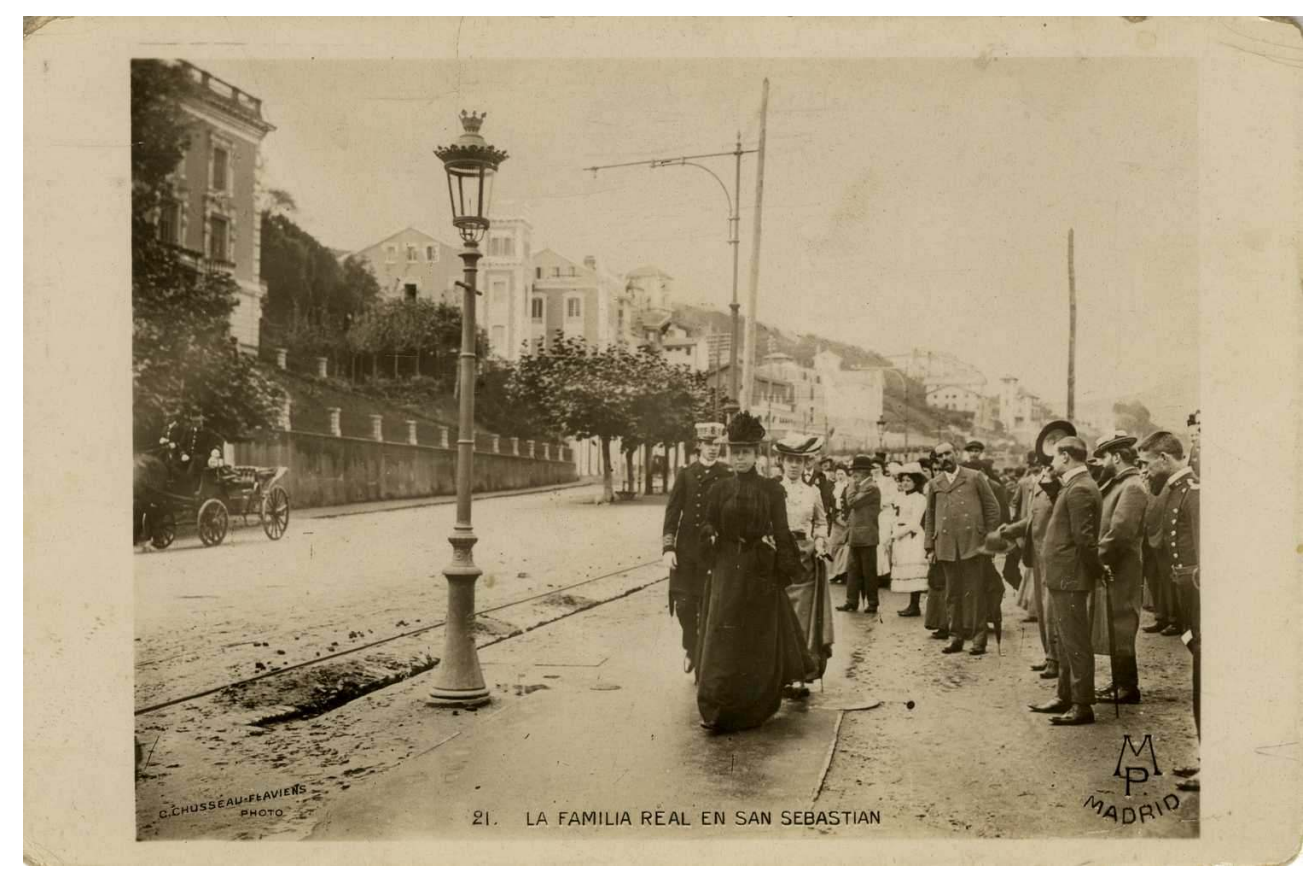

Photographie de C. Chusseau-Flaviens (réf. DMD0220048), www.guregipuzkoa.eus

\section{Une ville bourgeoise et cosmopolite}

Le cosmopolitisme était l'une des composantes de la ville réinventée. Saint-Sébastien devait se comparer à d'autres capitales touristiques européennes en termes de luxe et de confort, en projetant l'image d'une ville moderne, civilisée et bourgeoise, à même d'attirer les élites internationales. Pour ce faire, il était nécessaire de rompre avec les clichés associés au mythe romantique espagnol, en mettant l'accent sur la proximité géographique et culturelle de Saint-Sébastien avec l'Europe ${ }^{14}$. La presse locale insiste sur ce point depuis la fin du XIX $x^{e}$ siècle :

11 No existe en España población más bella, más nueva, más limpia, más sana. Luego, ¡hay que confesarlo! su proximidad a la frontera de Francia la hace ser, naturalmente, la capital española más cercana a Europa, la Europa de nuestros ensueños, la del progreso portentoso en costumbres; y los aires europeos ejercen sobre ella su influjo, como los del mar le ejercen sobre los enfermos que buscan la playa. ${ }^{15}$

De même, les premiers guides de Saint-Sébastien, publiés par le Sindicato de Festejos (prédécesseur du Sindicato de Iniciativa y Propaganda créé en 1909), plaçaient la ville dans une enclave privilégiée, à quelques heures de distance des grandes capitales comme Madrid ou Paris. Ils louaient son urbanisation équilibrée ou ses normes d'hygiène et de confort qui en faisaient, selon les auteurs de ces guides, une ville moderne, comparable à d'autres destinations touristiques européennes à la mode :

Colocado a las puertas de Europa y a distancia de catorce horas de Madrid y once de París, en los trenes de lujo; su playa disputa con éxito la predilección del mundo elegante. Ciudad más hermosa, mejor cuidada, con todo género de comodidades y sus servicios de higiene con arreglo al progreso moderno, no es posible que exista... El extranjero que la visita por primera vez, aunque tenga referencias de su fama y 
reputación, queda atónito y sorprendido. Al penetrar en la ciudad crece su admiración y no se cansa de observar la amplitud de sus calles, la belleza de sus monumentos, los lindos parques, jardines y paseos, y la variedad de las construcciones con una limpieza extremada en todo. ${ }^{16}$

Le cosmopolitisme à Saint-Sébastien repose sur des politiques de modernisation promues par les autorités locales, souvent avec l'aide du conseil provincial. Ainsi, par exemple, l'éclairage public électrique fut inauguré en 1899 dans les rues de la ville; en 1903, le service de tramway; le réseau téléphonique local, à son tour, fonctionne depuis 1908. Toutes ces avancées sont accompagnées de mesures visant à améliorer l'hygiène publique et l'assainissement des zones urbaines, depuis le réseau de traitement des eaux jusqu'à l'organisation d'un système municipal de protection sociale en faveur des classes pauvres de la ville. Ces politiques municipales, en outre, tout en cherchant l'amélioration de la qualité de vie des habitants, tendent à évincer de l'espace public toute expression de pauvreté ou de conflit social qui pourrait ternir l'image de la ville.

La topographie de la capitale bourgeoise et cosmopolite que les autorités locales ont essayé de promouvoir était principalement concentrée dans les espaces de l'expansion urbaine, avec ses larges avenues et boulevards, ses hôtels de luxe et ses bâtiments remarquables, parmi lesquels le Casino, inauguré en 1887 (voir illustration ci-dessous).

\section{Gran Casino de Saint-Sébastién (circa 1920)}

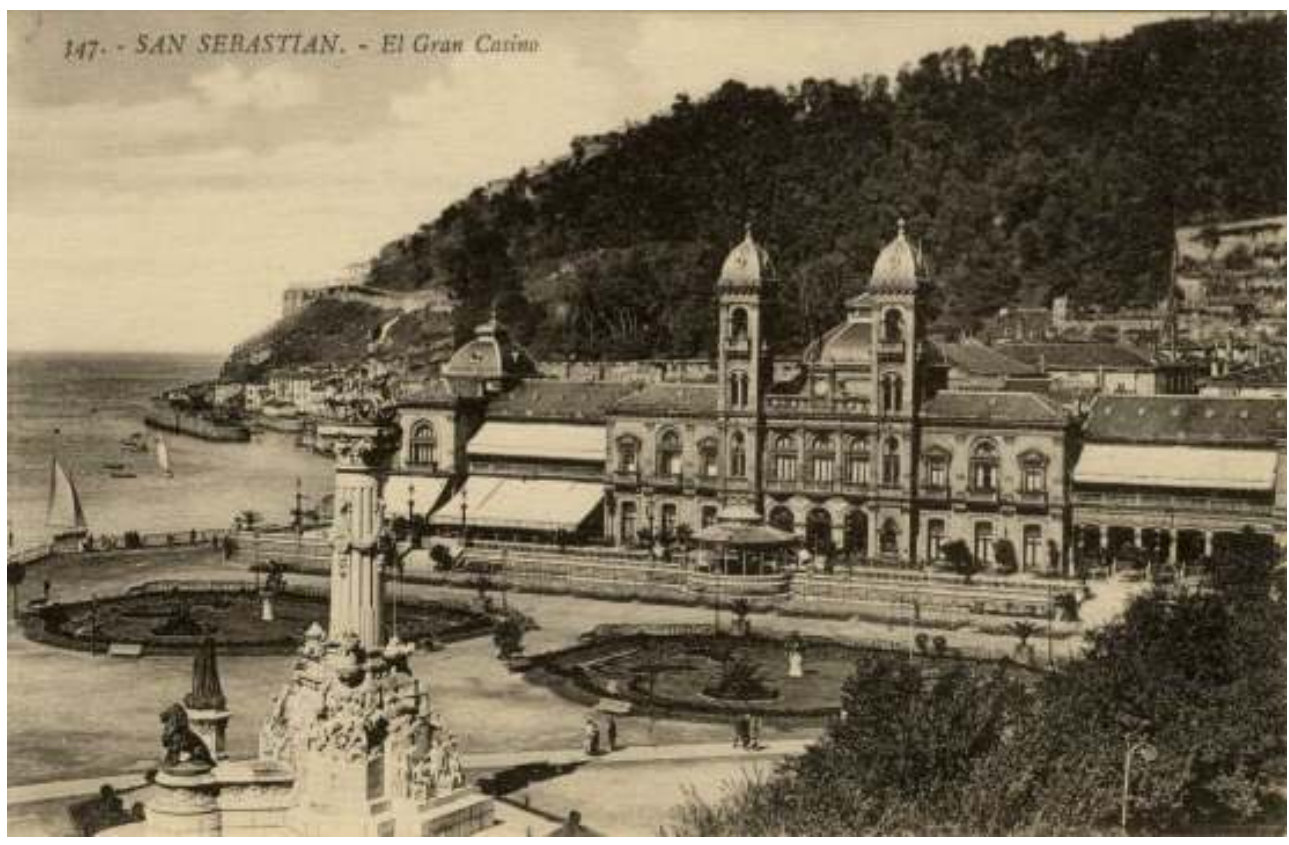

Carte postale (Gregorio G. Galarza ed.) ; réf. : DMD0060013, www.guregipuzkoa.eus

Le Casino, avec le boulevard et le Paseo de La Concha, complète la topographie urbaine identifiée au tourisme de luxe. Une publication du début $\mathrm{du} \mathrm{xx}^{\mathrm{e}}$ siècle venait à souligner l'importance du casino en tant qu'emblème de la ville moderne et cosmopolite que SaintSébastien entendait devenir, en s'inspirant largement de sa voisine Biarritz :

Se levanta este magnífico palacio, que nosotros lo denominamos de las Fiestas, en un extremo del antiguo campo de maniobras frente al Boulevard, el parque Alderdieder y a la Concha. Ningún establecimiento de este género en el extranjero reúne mejores condiciones que el Casino de San Sebastián. Encerrado en un ancho perímetro de 800 metros cuadrados, en los cuales se comprenden el emplazamiento 
del edificio y los jardines anexos, dominando toda la Concha y el Boulevard, y rodeado de elegante verja, resulta un conjunto en el que se harmonizan [sic] preciosamente las bellezas arquitectónicas con su situación ideal ${ }^{17}$

Ces espaces exclusifs abritaient également les principales activités de loisirs et d'agrément, tant le jour que la nuit, qui occupaient les vacanciers distingués, avec la famille royale en tête. Ainsi, par exemple, au Casino furent organisés des concerts, des cotillons, des galas de charité ou des feux d'artifice, pour le plaisir d'un public composé notamment de classes aisées, de la haute aristocratie ou des membres de la classe politique et diplomatique accompagnant les rois dans leur cour d'été. Outre l'effet de prestige et de propagande que de telles activités pouvaient avoir sur la ville, elles ont eu un impact positif avéré sur l'économie locale. Ce qui explique la décision, prise en 1905 avec l'accord des autorités locales, de prolonger l'ouverture du Casino au-delà de la saison estivale :

El importante centro de recreos, o suntuoso "Palacio de las Fiestas" ha cumplido desde que se inauguró los fines que de él esperaba la población al crearlo; y siguiendo con generoso empeño su misión de contribuir al progreso y engrandecimiento de San Sebastián y al fomento de sus intereses materiales, ha decidido desde éste (1905), continuar abierto todo el año, como poderosa y eficaz atracción para los turistas extranjeros que durante el invierno y la primavera recorren las poblaciones donde encuentran cómodo y confortable albergue, gratas distracciones y suma de facilidades para disfrutar los caprichos que les permite satisfacer su dinero. ${ }^{18}$

La création d'enclaves urbaines pour le tourisme de luxe a généré en retour des dynamiques de ségrégation socio-spatiale. La construction de cabines de bain sur la plage de La Concha en discriminant les baigneurs par sexe, lieu de baignade ou classe sociale, en constitue un bon exemple ${ }^{19}$. Tout comme la transformation progressive de la promenade du Boulevard, autrefois fréquentée par les classes populaires, en un «boulevard de style parisien », parsemé de "boutiques de luxe » et d'«élégantes vitrines » réservées aux touristes et aux élites locales ${ }^{20}$. 


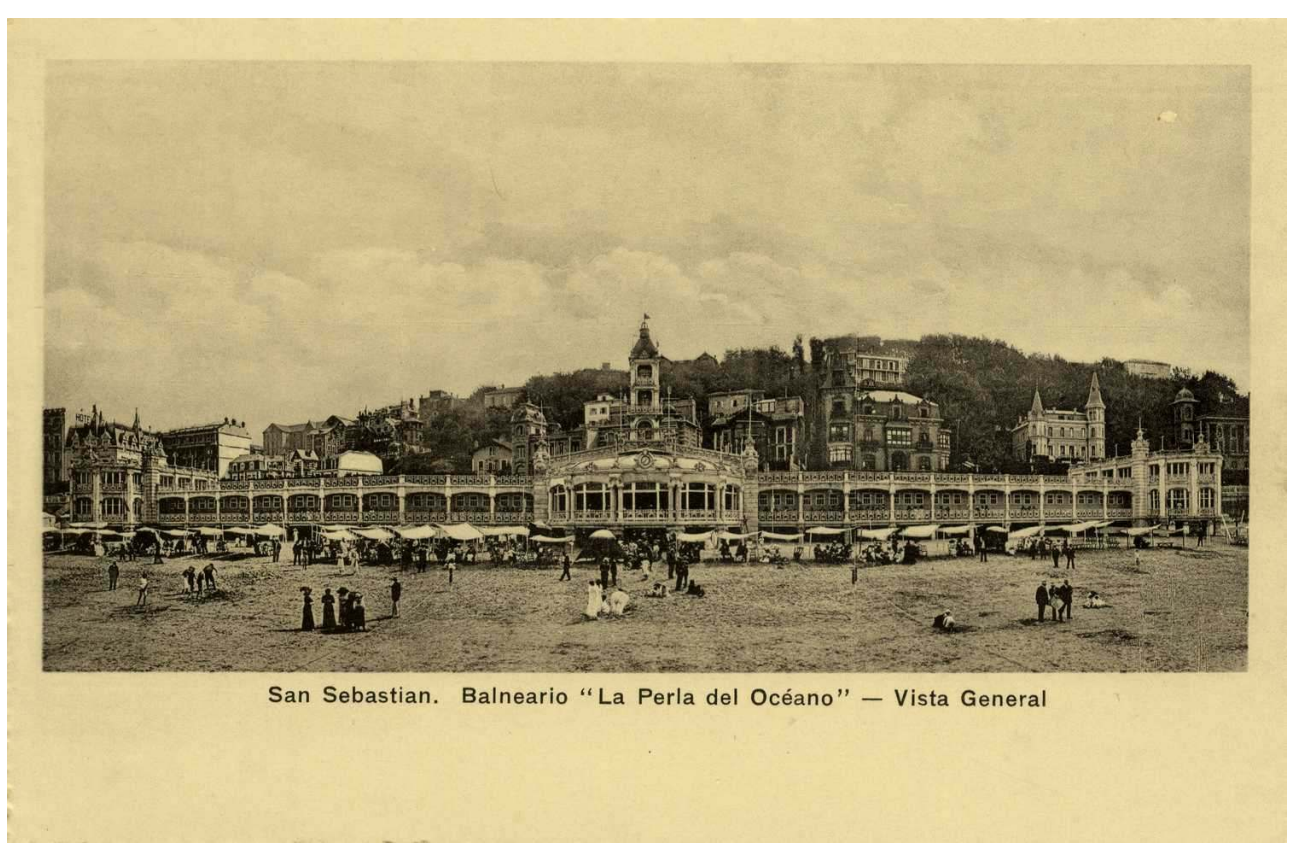

Cliché Willy Koch ; réf. : DMD0070256, www.guregipuzkoa.eus

Or Saint-Sébastien n'était pas seulement un espace urbain équilibré et harmonieux, comparable à celui d'autres capitales touristiques européennes. La ville se situait en plus dans une enclave privilégiée où convergeaient le paysage du littoral et le paysage alpin.

\section{Où la mer et la montagne s'embrassent}

19 Si nous analysons l'iconographie du premier tourisme basque, nous verrons que l'image de Saint-Sébastien affichée par les différents guides et brochures touristiques a moins trait aux espaces urbains qu'à l'environnement naturel de la ville. Un environnement qui combinait les avantages esthétiques et thérapeutiques que les représentations de l'époque attribuaient à la mer et à la montagne. L'un des premiers guides de Saint Sébastien, publié en 1907 par le Sindicato Municipal de Festejos, l'exprimait ainsi :

El clima de San Sebastián es a la vez agradable y sano ; agradable porque ni el calor es intenso, ni los fríos son grandes; sano porque no hay cambios bruscos de temperatura y siempre se respira o el aire del mar o el de la montaña. ${ }^{21}$

Tout d'abord, force est de constater que les pratiques touristiques liées aux bains de mer ont contribué à déplacer l'épicentre urbain vers la côte jusqu'à ce que la plage de $L a$ Concha devienne le symbole principal de Saint-Sébastien. En fait, cette plage et la promenade homonyme figurent parmi les principaux attraits touristiques de la ville comme en témoignent les différents livres de voyage et guides touristiques que nous avons pu consulter. La description faite en 1886 par Alfredo Laffitte, journaliste et homme politique de Saint Sébastien, surprend par son expressivité :

La Concha en sus tres fases de paseo, playa y bahía, entretiene la animación del verano; como paseo puede muy bien competir con la célebre corniche de Niza. Lindos hotelitos de familias, hermosas fondas, chalets en la falda del trozo de carretera que va al Antiguo, piso excelente y pretil, desde el que se contempla cual de un espacioso mirador la gente que se baña, los buques que entran, los soberbios 
montes que cierran la bahía, y el horizonte inmenso de alta mar. Este paseo debiera llamarse de España, porque por él atraviesan individuos de las cuarenta y nueve provincias de la Península. Como playa es un encanto; es la más cómoda, limpia, segura y proporcionada que se encuentra entre las de su clase, nacionales y extranjeras. Con su elegante forma de Concha, rodeando la población... tiene un bien montado establecimiento de baños denominado la Perla del Océano e infinidad de casetas [...] ¡Qué animación, qué algazara !22

21 Par ailleurs, en parcourant les promenades comme La Concha les touristes pouvaient apprécier les beautés "naturelles» de Saint-Sébastien tout en jouissant d'une vue exceptionnelle sur la mer. Pour cette raison, ces promenades figuraient parmi les itinéraires recommandés par les guides, comme complément indissociable des bains de mer et des activités de loisirs qu'offrait la ville ${ }^{23}$. Ce n'est pas par hasard que, comme le rappelle Alain Corbin à juste titre, la vue sur la mer a conféré depuis la fin du XVIII ${ }^{\mathrm{e}}$ siècle une valeur ajoutée indiscutable à toute ville à vocation thermale ${ }^{24}$.

L'iconographie de l'ensemble de la promenade et de la plage de La Concha, reproduite à satiété dans des cartes postales, guides, affiches touristiques, couvertures de magazines, etc. finira par placer cet espace au cœur d'un paysage qui a fait de Saint-Sébastien un paradis touristique. Le Guide officiel de Saint-Sébastien de 1917-1918, par exemple, offrait un portrait précis de cet ensemble :

(la playa de La Concha) es la más bella que pueda existir en el mundo. Tiene, como su nombre lo indica, la forma exacta y graciosa de una inmensa concha. La elegante perfección de su línea, la finura de sus arenas, la regularidad y dulzura habituales de sus olas, que antes de venir a expirar sobre la orilla hánse amortiguado en el rompeolas natural de la isla de Santa Clara; el admirable panorama que se extiende ante nuestra vista, todo contribuye a maravillar y cautivar al espectador. A los encantos naturales de este panorama único, la mano del hombre ha añadido algo: un paseo magnífico sobre la playa que domina todo lo largo de la Concha, circundado de frondosos tamarindos que dan protectora sombra. Un magnífico voladizo o paseo cubierto a la orilla del mar que se extiende alrededor de toda la playa sirve de refugio a los bañistas los días lluviosos. Y sirviendo de marco a todas estas bellezas una serie de villas y hoteles suntuosos, cuyas fachadas y jardines dan la nota alegre de este hermoso paseo. ${ }^{25}$ 
La Concha - Promenade et plage - Carte postale

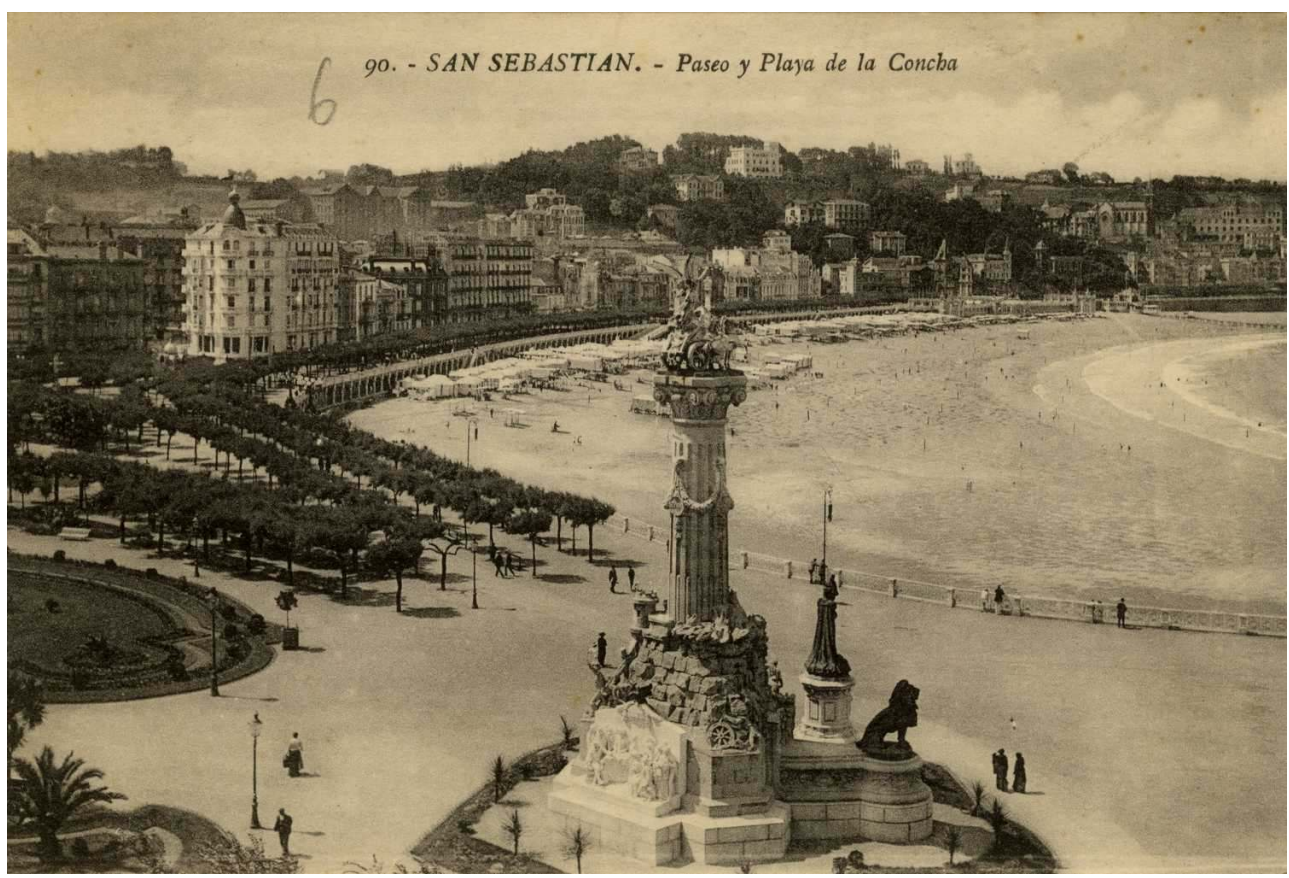

Éditeur : Gregorio G. Galarza (non daté), probablement entre 1913 et 192026. Réf. : DMD0060001 ; www.guregipuzkoa.eus

Les sports maritimes associés au tourisme, promus principalement par le roi Alphonse XIII, ont également contribué à changer le regard porté sur les zones côtières ${ }^{27}$. Les courses internationales de bateaux, les compétitions de chalutiers, les festivités nautiques tenues dans la baie de La Concha ou sur la rivière Urumea, occupaient en effet une place grandissante dans le programme des fêtes de la période estivale (voir illustration cidessous $)^{28}$. 


\section{El lestival del domingo en el Club Náutico}

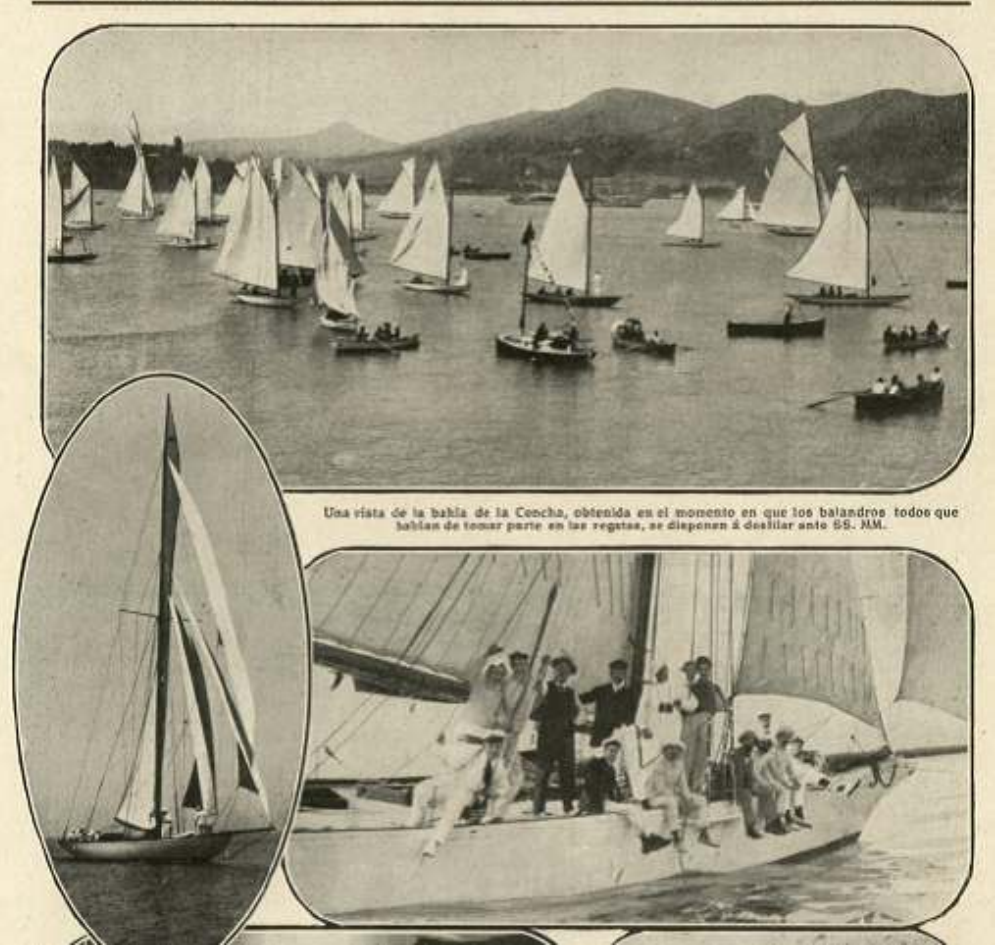

Revue Novedades, 16 juillet 1911.

Ces événements sportifs attiraient l'attention non seulement des touristes et des vacanciers, mais aussi de la population locale et provinciale. Les sources de l'époque en sont témoins :

Pendant l'été (à Saint-Sébastien) ont lieu sans interruption des régates de yachts nationales et internationales organisées par le Real Club Náutico, ainsi que des régates de yoles. Durant le mois de septembre sous le patronage de l'Ayuntamiento de SaintSébastien et du Club Marítimo se célèbrent les fameuses régates de traînières montées par les pêcheurs de la côte basque. Ce spectacle attire la population côtière de Guipuzcoa. ${ }^{29}$

De la sorte, les représentations associées à la mer Cantabrique commencent à changer. Cette mer, perçue jadis comme un territoire sauvage et dangereux, milieu difficile dans lequel se forgeaient des marins courageux et où périssaient des pécheurs, devient progressivement un espace de loisirs et d'agrément ${ }^{30}$. Les pratiques touristiques ont ainsi mené à un autre type d'appropriation du littoral, en faisant de la mer une composante à part entière du paysage urbain.

Toutefois, Saint-Sébastien ne s'ouvrait pas seulement sur la mer. La ville était aussi entourée de collines et de montagnes, dans un environnement que la sensibilité romantique associait aux paysages alpins ${ }^{31}$. En effet, les collines environnantes font progressivement partie du périmètre urbain, à la fois par la création d'infrastructures qui les relient au centre-ville (navette pour gagner le mont Ulia inaugurée en 1902; funiculaire du mont Igueldo, mise en place en 1912) que par les pratiques de randonnée de plus en plus populaires, qui transforment ces espaces naturels en zones de loisirs destinés tant aux touristes qu'à la population locale. De plus, à partir de ces 
promontoires, les randonneurs pouvaient jouir des vues « les plus splendides » de la ville et de ses environs ${ }^{32}$.

Vue de Saint-Sébastien depuis le mont Igueldo, 1929

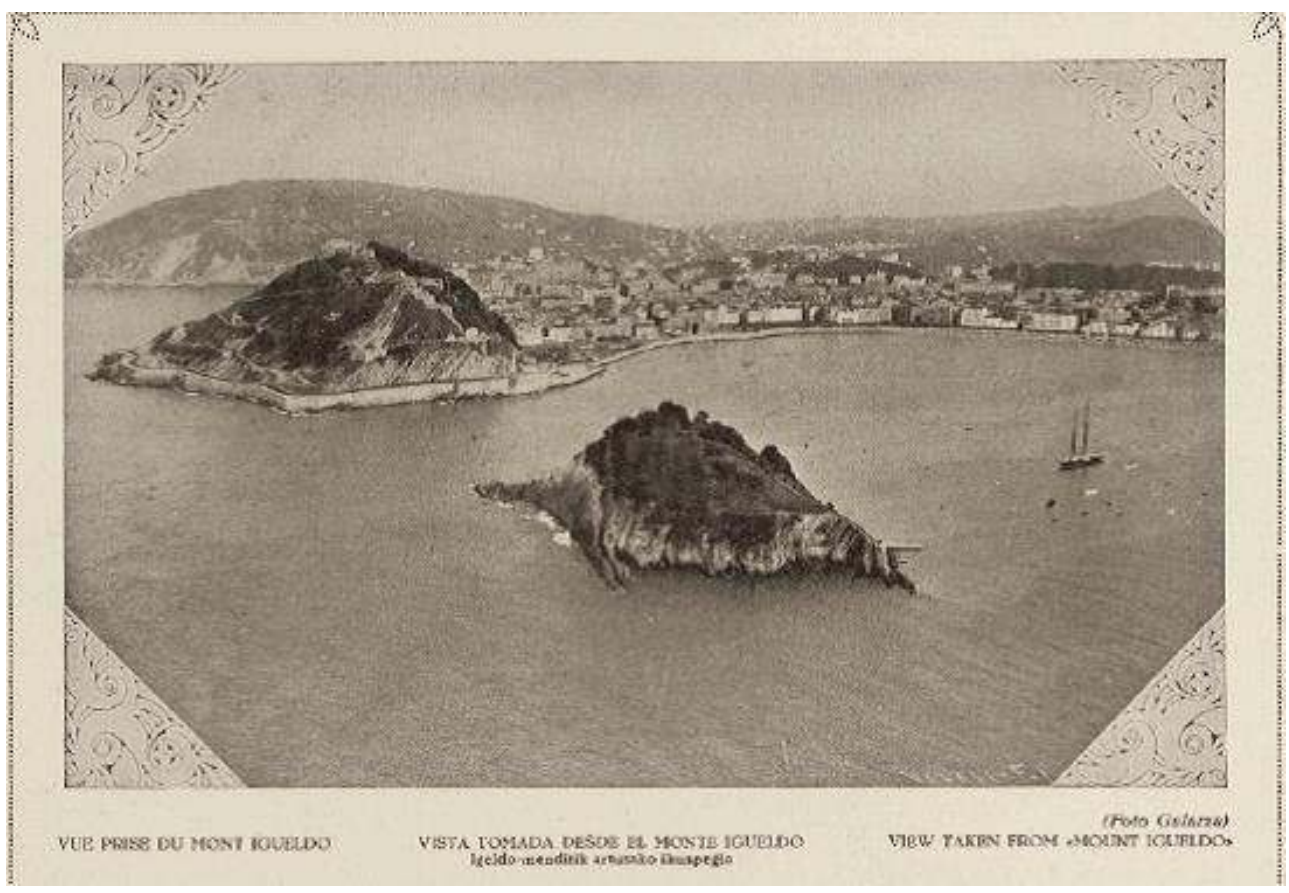

Guipúzcoa deportiva y aristocrática, été-automne 1929 (Sur la photo, I'on peut apercevoir la baie de La Concha, l'île de Santa Clara, le Mont Urgull et le Mont Ulia)

Ainsi, la confluence de la mer et de la montagne, avec les avantages associés aux paysages côtiers et alpins respectivement, a fait de Saint-Sébastien une destination touristique privilégiée, avec une claire vocation internationale ${ }^{33}$. Un article paru dans un journal vers la fin des années 1920, intitulé « San Sebastián. Mar y Montaña » (Saint Sébastien. Mer et Montagne) résumait bien cette idée :

\begin{abstract}
Mar y Montaña, contraste atrevido, que pueden realizar pocas playas y que es, sin embargo, la característica de San Sebastián... Al mar vivificante, a las excitaciones del aire salino, se añaden las delicias de las pintorescas excursiones a los alrededores y sobre todo los paseos, que, sin cansancio, por cuestas suaves, os llevan a las cimas del Monte Igueldo, del Monte Urgull y del Monte Ulía, entre los abetos de balsámicos efluvios, beneficiosos a los pulmones delicados. Para los ascensionistas, alrededor y al lado, están los Pirineos, de cimas abruptas y tentadoras... San Sebastián, llamada a justo título la Perla del Océano, muestra al viajero excepcionales contrastes que son la base de su extraordinario poder sugestivo. ${ }^{34}$
\end{abstract}

\title{
Une ville pittoresque
}

L'image de Saint-Sébastien comme ville moderne et cosmopolite, forgée par les autorités locales et les différents agents associés au tourisme, avait son contrepoint dans le goût pour le pittoresque. En fait, depuis la fin du XIx ${ }^{\mathrm{e}}$ siècle, le «typisme » de la capitale basque a servi pour attirer des touristes nationaux et étrangers. Nous en avons un exemple dans les corridas de taureaux, conçues par l'imaginaire romantique, surtout français, come expression de l'« exotisme » espagnol. Malgré les réticences des autorités locales envers 
le spectacle taurin ${ }^{35}$, quitte à ternir l'image bourgeoise et cosmopolite de Saint-Sébastien, la corrida devint l'un des principaux atouts touristiques de Saint-Sébastien, en procurant à la ville des avantages économiques aussi bien que «patriotiques $»^{36}$. La presse de l'époque le reconnaissait ouvertement :

Las corridas de toros siguen siendo en San Sebastián un elemento insustituible de animación y de vida... A presenciar las corridas vienen a San Sebastián algunos millares de franceses que dejan su dinero, no solamente en la taquilla de la plaza, sino también en las del ferrocarril, en fondas, restaurants, comercios, tranvías, cafés, etc. Y véase por dónde, esta empresa taurina, merced a su acierto en la organización del espectáculo, desarrolla una labor patriótica, aumentando la corriente de turistas franceses que de este modo van tomando afición a las cosas de España. ${ }^{37}$

Le spectacle de la tauromachie a été par ailleurs épaulé par la construction en 1903 d'une arène située dans le quartier de Gros, à l'est de la ville. Connu sous le nom de place du Chofre, elle avait une capacité d'accueil de 13000 spectateurs.

Les manifestations liées à l'identité locale avaient aussi leurs propres espaces: des tavernes et des sociétés populaires, concentrées principalement dans la vielle ville et dans les quartiers périphériques, comme celui de $\mathrm{El}$ Antiguo, des frontons de pelote basque (voir illustration ci-dessous), ou encore des pèlerinages (romerías) organisés dans les zones rurales proches (telles que Loyola ou Eguía). Ces espaces permettaient aux vacanciers illustres de jouir en toute sécurité du contact direct avec le "pittoresque», souvent compris comme synonyme de populaire. Les romerías de la vallée de Loyola, par exemple, s'organisaient chaque été vers la fin juillet et sont devenues une des principales attractions du programme estival. Voici comment le chroniqueur Luis de León décrit le romería de 1905 :

En cordial confusión de clases, los romeros salen de San Sebastián a media tarde, embarcados en viejas lanchas y gabarras... El río aparece lleno de embarcaciones cargadas de mujeres elegantes, ataviadas con vistosos y delicados trajes de verano. En el fondo de los barquichuelos van las vituallas que van a consumirse en el escondido valle de Loyola.

La subida tiene cierto aspecto de fiesta oficial. En una barca van los miembros del Ayuntamiento; en otra, los políticos de banda... En una enorme gabarra navegan los orfeonistas; el montón de boinas rojas contrasta vivamente con el tono verde del paisaje. La banda municipal marcha en otro pesado gabarrón...

Ya en el valle de Loyola, cada grupo de turistas ocupa una tribuna o una carga; otros siguen en las embarcaciones, bajo la sombra de los sauces. Y todo el mundo se pone a merendar. En las riberas inmediatas, el pueblo se reúne en corros. A pesar de esta confusión de clases, no hay una sola nota chocarrera o de mal gusto. El pueblo, este buen pueblo guipuzcoano, compite en suaves maneras con la propia aristocracia. El refinamiento y la cultura son cualidades ingénitas de su espíritu. ${ }^{38}$

Un autre des espaces typiques que les guides recommandaient de visiter était le port des pêcheurs, jouxtant la vieille ville. Les touristes pouvaient y trouver une atmosphère "vivante et pittoresque ", composée des bateaux de pêche qui revenaient "empaquetés de sardines » ou des « aguerries pêcheuses de Saint-Sébastien " ${ }^{39}$.

Les manifestations du typisme local se sont renforcées à partir du début du $\mathrm{Xx}^{\mathrm{e}}$ siècle, coïncidant avec la montée du basquisme. Il s'agit d'un vaste mouvement culturel né à la fin $\mathrm{du} \mathrm{XIX}^{\mathrm{e}}$ siècle, dans lequel convergent des groupes politiques distincts (carlistes, partisans des fueros, régionalistes, nationalistes basques). Son but était de promouvoir, par l'organisation de diverses activités, la langue, le folklore ou les traditions relevant du particularisme basque. 
Fronton de pelote Jai-Alai, Saint-Sébastien, 1917

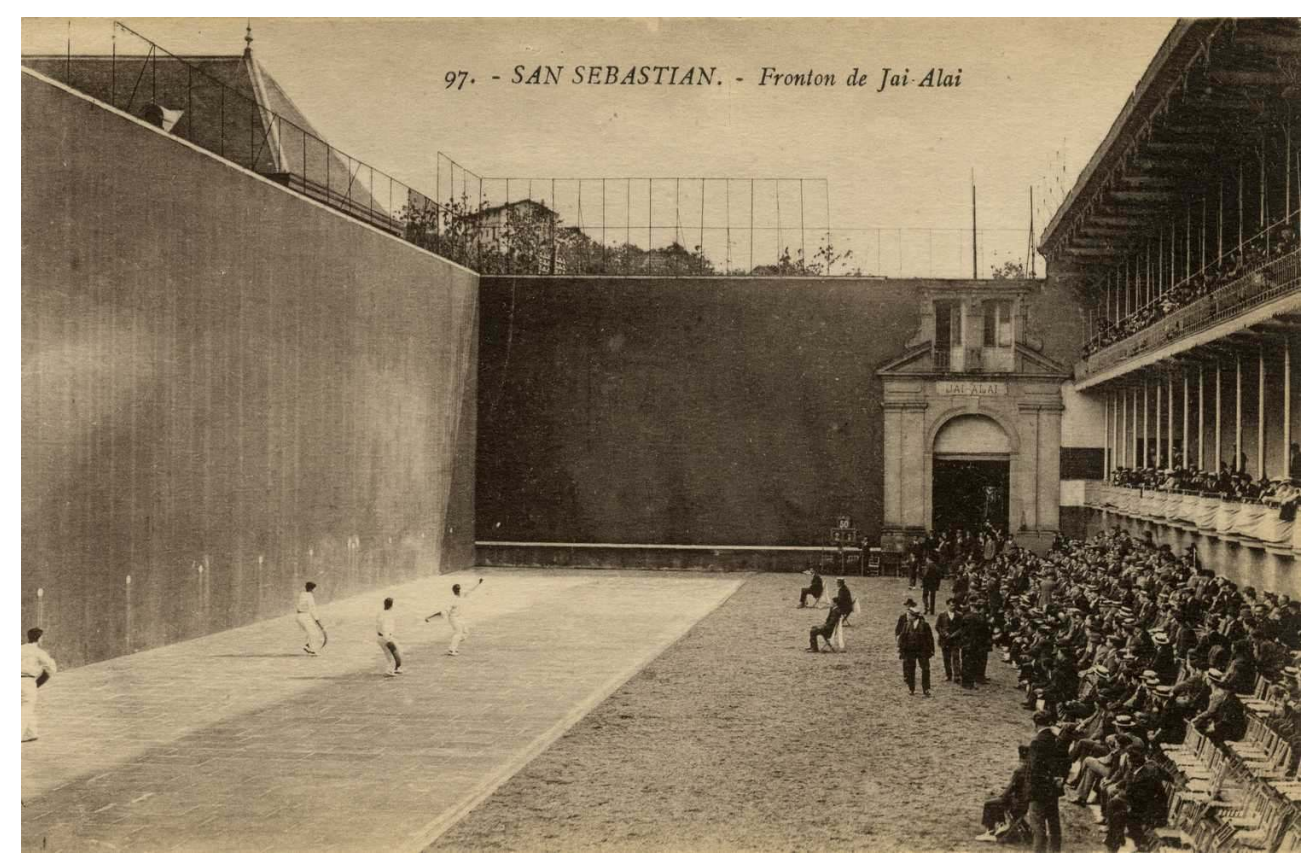

Carte postale éditée par Gregorio G. Galarza, 1917. Fototeka Kutxa-réf. : KUTXA_MACA_1_0282_E-459 www.guregipuzkoa.eus

Ce particularisme pouvait cependant obéir à d'autres intérêts, dans la mesure où il devient une ressource touristique, un atout majeur pour Saint-Sébastien. À l'approche des années 1920, le goût pour le " pittoresque " gagne en force comme carte de visite de la ville, en se superposant à ses charmes naturels ou à son luxe et confort modernes ${ }^{40}$. L'iconographie ethnique s'insère progressivement dans les affiches publicitaires et les guides touristiques de Saint-Sébastien. Les pages des guides se voient illustrées par des paysages et des "scènes basques" pour répondre à la demande d'un tourisme à la recherche d'authenticitét ${ }^{41}$. De même, de nouveaux itinéraires sont conçus pour permettre aux touristes et vacanciers de connaître le patrimoine de la région ou d'entrer en contact avec les traditions locales. 


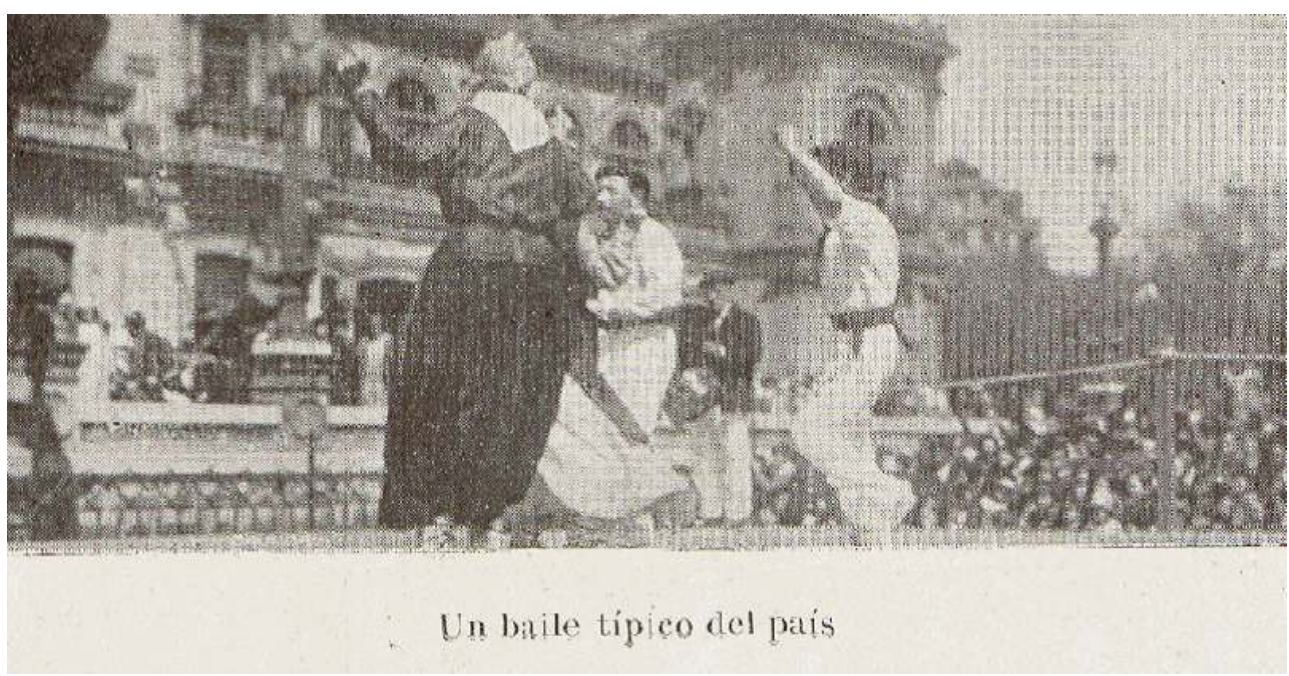

Illustration publiée dans Bellezas de San Sebastián y sus alrededores, Saint Sébastien, Leizaola Typographie, 1929.

\section{En guise de conclusion}

Comme nous avons pu le constater, la géographie est activement impliquée dans les processus historiques. Les espaces géographiques, en l'occurrence urbains, sont redéfinis en permanence en fonction des représentations et des pratiques des groupes humains qui les habitent. Comme le souligne Augustin Berque, «les sociétés organisent leur environnement comme elles le perçoivent et elles perçoivent leur environnement comme elles l'organisent $»^{42}$. Dans l'exemple de Saint-Sébastien analysé ici nous avons montré que le tourisme et les pratiques sociales qui lui sont associées ont contribué à redéfinir la ville et à en faire une destination touristique de référence internationale. Mais ils ont aussi eu un impact, par la construction culturelle d'un nouveau paysage urbain, sur les dynamiques identitaires déclenchées dans la région par le processus d'industrialisation. Le cosmopolitisme en tant que marqueur identitaire de Saint-Sébastien, mis en valeur par les différents agents touristiques, interagira avec d'autres identités collectives qui se sont produites au niveau local - sociales, culturelles ou territoriales -, parfois favorisées par le tourisme, parfois transformées par lui. La création des parcours urbains exclusifs et inclusifs dans la ville, aménagés par le tourisme, le lien étroit entre la capitale et son environnement rural ou encore l'intégration progressive de la mer et de la montagne dans l'espace urbain, sont des exemples montrant comment le paysage n'est pas un simple décor des processus sociaux mais en est un élément constitutif. 


\section{NOTES}

1. Angelo Torre, "Un "tournant spatial" en histoire? Paysages, regards, ressources ", Annales. Histoire, Sciences Sociales, nº 5, 2008, p. 1127-1144.

2. "Saint-Sébastien aux origines du tourisme basque", communication présentée dans le colloque international "La ville dans le monde hispanique. Espace(s) et représentation(s)», organisé par Christine Delfour et Emmanuel Vincenot, Université Paris-Est Marne-La-Vallée, 14 et 15 janvier 2016.

3. Pour une approche du tourisme depuis la perspective de l'histoire économique: Carlos Larrinaga, Balnearios guipuzcoanos, 1776-1901. Turismo de salud e inversión de capital en aguas minerales, San Sebastián, Real Sociedad Bascongada de Amigos del País, 2014.

4. Marc Boyer, Histoire générale du tourisme. Du XVI a a XXI siècle, Paris, L'Harmattan, 2005.

5. Ana Moreno Garrido, Historia del turismo en España en el siglo XX, Madrid, Síntesis, 2007, p. 12.

6. Pour une relation entre agentivité et pratiques spatiales, bien qu'en se référant à la formation de la classe ouvrière anglaise, l'on peut consulter : David Featherstone et Paul Griffin, "Spacial relations, histories from below and the makings of agency: Reflections on The Making of the English Working Class at 50", Progress in Human Geography, vol. 40(3), 2016, p. 375-393.

7. John K. Walton et Jenny Smith, "The First Century of Beach Tourism in Spain: San Sebastian and the Playas del Norte from the 1830s to the 1930s", dans Michael Barke, John Towner y Michael T. Newton, Tourism in Spain. Critical Issues, Oxon (UK), Cab International, 1996, p. 35-61.

8. Selon C. Larrinaga, en 1877 , la plus forte concentration de sources minérales par kilomètre carré en Espagne se trouvait dans les provinces de Guipúzcoa et de Vizcaya. En outre, ces provinces avaient les stations thermales les plus prestigieuses. (Carlos Larrinaga, op. cit., p. 8).

9. La revue basque Euskal-Erria rapportait en 1895 l'inauguration d'un nouveau Grand Hôtel « construit avec toutes les avancées modernes » autour du balnéaire de Cestona dans la province de Guipuzcoa (Euskal-Erria, t. 32, $1^{\mathrm{er}}$ semestre, 1895), p. 535.

10. La sensibilité romantique du XIX ${ }^{\mathrm{e}}$ siècle favorise la "découverte " de la côte comme lieu de loisir et objet de contemplation (Alain Corbin, Le territoire du vide. L'Occident et le désir du rivage, Paris, Éditions Flammarion, 1990, $1^{\text {a }}$ ed. 1988).

11. Carlos Larrinaga, "De las playas frías a las playas templadas: la popularización del turismo de ola en la España del siglo XX”, Cuadernos de Historia Contemporánea, vol. 37 (2015), p. 67-87.

12. Sur l'importance du tourisme dans la croissance et le développement de Saint-Sébastien: Luis Castells, « La Bella Easo: 1864-1936 », dans Miguel Artola (ed.), Historia de Donostia-San Sebastián, San Sebastián, Nerea, 2000, p. 283-386.

13. Laurent Tissot emploie le concept «système touristique" pour englober toutes les institutions, infrastructures et activités qui font d'une zone géographique donnée un "paradis touristique » (Laurent Tissot, "From Alpine Tourism to the 'Alpinization' of Tourism", dans Eric G.E. Zuelow (ed.), Touring Beyond the Nation. A Transnational Approach to European Tourism History, Burlington (USA), Ashgate, 2010, p. 59-78).

14. Pour une approche de la construction du mythe romantique espagnol du point de vue de l'imagologie, l'on peut consulter : Xavier Andreu-Miralles, "Diálogos asimétricos. Una propuesta de análisis de la imagen romántica de España”, Amnis [En ligne], 2018, mis en ligne le 10 mars 2018, consulté le 22 mai 2018. URL : http://journals.openedition.org/amnis/3254.

15. "El veraneo en San Sebastián", Euskal Erria, t. 49 (2e semestre 1903), p. 63.

16. San Sebastián, verano 1907 (Archives municipales de Saint-Sébastien, B-7-VI). 
17. Luis de León, “San Sebastián. Crónica del verano de 1905”, p. 2 (Archives municipales de Saint-Sébastien, B-7-VI, 275-10).

18. Luis de León, op. cit., p. 5.

19. David Mota Zurdo, "Costa vasca", dans Santiago de Pablo (Coord.), 100 Símbolos Vascos. Identidad, cultura, nacionalismo, Madrid, Tecnos, 2016, p. 92.

20. San Sebastián y sus alrededores, 1920, San Sebastián, Librería Internacional, 1920, p. 99.

21. "San Sebastián. Programa de Fiestas. Verano de 1907" (Archives municipales de SaintSébastien, B-7-VI).

22. Alfredo Laffitte, Tierra Euskara. Excursiones, cuadros y notas de Guipúzcoa, Tolosa, Imprenta y Casa Editorial de E. López, 1886, p. 184-186.

23. Un guide de Saint-Sébastian de 1898, par exemple, a compté jusqu'à 15 promenades, parmi lesquelles le Paseo de la Concha, en soulignant que celle-ci était « la plus favorisée par la colonie d'été ». (San Sebastián y sus alrededores. Guía ilustrada por Z. Leizaola, San Sebastián, J. Baroja e Hijo eds., 1898, p. 97).

24. Corbin, Alain, op. cit., p. 167 y ss.

25. San Sebastián. Guía oficial editada por el Sindicato de Iniciativa y Propaganda, 1917-1918 (guide gratuit), Archives-Bibliothèque Koldo Mitxelena de Saint-Sébastien.

26. Au premier plan, le monument du centenaire de Saint Sébastien, érigé en 1913 (il a été déplacé des jardins d'Alderdi-Eder au début des années 1920).

27. La passion d'Alfonso XIII pour la voile l'a amené à participer à de nombreuses régates organisées à Saint-Sébastien par le Royal Yacht Club (Félix Luengo Teixidor et Ander Delgado Cendagortagalarza, “Antes patria que rey. Los viajes de Alfonso XIII por el País Vasco y Navarra”, dans Margarita Barral Martínez (ed.), Alfonso XIII visita España. Monarquía y nación, Granada, Comares, 2015, p. 80-81).

28. Les journaux locaux, comme El Pueblo Vasco (1903-1936) ou le magazine Novedades (1909-1919), informaient largement sur les différentes activités touristiques.

29. San Sebastián y sus alrededores (Saint-Sébastien et ses environs), San Sebastián, Librería Internacional, 1920, p. 215.

30. Maitane Ostolaza, "Mar y montaña”, dans Santiago de Pablo (Coord.), 100 Símbolos Vascos. Identidad, cultura, nacionalismo, Madrid, Tecnos, 2016, p. 88-91.

31. Pour approfondir les caractéristiques du modèle paysagère alpin : Laurent Tissot, "From Alpine Tourism to the 'Alpinization' of Tourism”, dans Eric G.E. Zuelow (ed.), op. cit., pp. 59-78.

32. Des guides spécialisés informaient des effets bénéfiques qui pour les touristes et randonneurs avaient l'ascension aux montagnes situées aux abords de la ville: Epifanio G. Serrano, San Sebastián en la Mano. Monte Ulía, San Sebastián, Imprenta de Hijos de J. Baroja, 1914; du même auteur : San Sebastián en la Mano. Monte Igueldo, San Sebastián, Imprenta de Hijos de J. Baroja, 1914 (Archives Genérales de Guipúzcoa, Tolosa, JDSM-24).

33. L'internationalisation de Saint-Sébastien en tant que destination touristique a reçu une impulsion significative dans les années de la Première Guerre mondiale, lorsque la ville est devenue un refuge pour les aristocrates et les diplomates, en particulier français, qui ont fui la guerre (Félix Luengo, San Sebastián. La vida cotidiana de una ciudad, Donostia, Txertoa, 1999). Pour plus d'informations sur l'évolution du mouvement des « étrangers » à Saint-Sébastien, voir : Alet Valero, "El turismo de playa en España entre 1850 y 1959. Creación, madurez y crisis", dans Francis Fourneau et Andrés García Lorca (eds.), Desarrollo regional y crisis del turismo en Andalucía, Madrid, Casa de Velázquez, 1994, p. 297-329.

34. "San Sebastián. Mar y Montaña”, Guipúzcoa deportiva y aristocrática, été-automne 1930.

35. Lorsque la Mairie de Saint-Sébastien a débattu du texte à inclure dans le Guide officiel de l'exposition internationale de Bordeaux de 1895 comme publicité de la ville, la Commission de Spectacles du Conseil Municipal a insisté pour que l'accent soit mis sur tout ce qui concerne «les promenades urbaines, les jardins, les rues, les plages et les industries » et que les références à la 
corrida soient minimisées (Lettre du $1^{\mathrm{er}}$ juin 1891, Archives municipales de Saint-Sébastien, B-7I-273-4).

36. Le rôle politique et nationalisateur du tourisme, encouragé par les autorités espagnoles dans les années 1910 et 1920, a été étudié par: Jorge Villaverde, “¿Motor o lastre nacional? La controvertida actuación del marqués de la Vega Inclán como Comisario Regio de Turismo (1911-1928)". Je remercie l'auteur de m'avoir facilité l'accès à ce travail inédit.

37. “San Sebastián, playa de sport", Gran vida. Revista ilustrada de sports, Año VI, no 63 (août 1908), p. 6-7.

38. Luis De Leon, op. cit., p. 12.

39. San Sebastián y sus alrededores, 1920, San Sebastián, Librería Internacional, 1920, p. 111-112.

40. Plusieurs auteurs s'accordent pour dire que la période de la dictature de Primo de Rivera (1923-1930) a marqué un tournant dans l'activité touristique de Saint-Sébastien, conséquence de la crise de l'après-guerre et de l'interdiction des jeux de casino par le dictateur en 1924. Voir, par exemple : John. K. Walton et Jenny Smith, op. cit., p. 48-51. Pour faire face à la baisse du nombre de touristes, les autorités locales ont intensifié leurs efforts de publicité, enrichi le programme des fêtes de la saison estivale, rénové certaines installations sportives, comme le circuit de course automobile à Lasarte (à quelques kilomètres de Saint-Sébastien) ou encore promu les courses de chevaux avec la création, à partir de 1922, d'importants prix internationaux (Actas del Sindicato de Iniciativa y Propaganda de San Sebastián, 1910-1925, Archives-Bibliothèque Koldo Mitxelena de Saint Sébastien).

41. Un guide de Saint-Sébastien de 1928, publié par l'office national du tourisme espagnol ( Patronato Nacional de Turismo), récemment créé, comprenait une photographie avec des scènes de pèlerinages et de danses basques, ainsi qu'une autre dans laquelle un groupe de femmes vêtues de peignes, de mantilles et de costumes andalous parcouraient les arènes du Chofre. Les deux images ont été montrées comme des exemples du charme «pittoresque » de Saint-Sébastien (San Sebastián. Playa y montaña, 1928, Patronato Nacional de Turismo (Archives-Bibliothèque Koldo Mitxelena de Saint-Sébastien).

42. "Les sociétés aménagent leur milieu comme elles le perçoivent et elles le perçoivent comme elles l'aménagent» (Augustin Berque, La mésologie. Pourquoi et pour quoi faire? Paris, Presses universitaires de Paris Ouest, 2014, p. 29).

\section{RÉSUMÉS}

Dans le contexte du «tournant spatial » que l'historiographie a connu ces dernières années, cet article propose une approche des espaces urbains et de leur évolution dans la perspective des études du paysage. À partir d'un cas historique spécifique, comme celui de la ville de SaintSébastien pendant la Restauration espagnole (1876-1931), il analyse les relations entre l'espace urbain, le paysage et les identités collectives à travers le développement du tourisme. Un phénomène social dont l'essor coïncide avec l'industrialisation de la région basque, entre la fin $\mathrm{du} \mathrm{XIX}^{\mathrm{e}}$ siècle et les premières décennies du Xx $\mathrm{x}^{\mathrm{e}}$ siècle.

En el contexto del "giro espacial" que ha conocido la historiografía en los últimos años, este artículo propone un acercamiento a los espacios urbanos y su evolución desde la perspectiva de los estudios de paisaje. A partir de un caso histórico concreto, como es el de la ciudad de San Sebastián durante la Restauración española (1876-1931), analiza las relaciones entre espacio 
urbano, paisaje e identidades colectivas a partir del desarrollo del turismo, como fenómeno social ligado a la industrialización en la que la región vasca se vio inmersa entre finales del siglo XIX y primeras décadas del XX.

INDEX

Mots-clés : paysage, espace urbain, tourisme, identités collectives, Saint Sébastien.

Palabras claves : paisaje, espacio urbano, turismo, identidades colectivas, San Sebastián.

\section{AUTEUR}

MAITANE OSTOLAZA

Sorbonne Université-Crimic 